\title{
Discrepant Effects of Human Interferon-gamma on Clinical and Immunological Disease Parameters in a Novel Marmoset Model for Multiple Sclerosis
}

\author{
S. Anwar Jagessar • Bruno Gran • Nicole Heijmans • \\ Jan Bauer • Jon D. Laman • Bert A. 't Hart • \\ Cris S. Constantinescu
}

Received: 16 August 2011 / Accepted: 2 October 2011 /Published online: 20 October 2011

(C) The Author(s) 2011. This article is published with open access at Springerlink.com

\begin{abstract}
The core pathogenic process in the common marmoset model of multiple sclerosis (MS) is the activation of memory-like $\mathrm{T}$ cells specific for peptide 34 to 56 derived from the extracellular domain of myelin/oligodendrocyte glycoprotein $\left(\mathrm{MOG}_{34-56}\right)$. Immunization with $\mathrm{MOG}_{34-56}$ in incomplete Freund's adjuvant is a sufficient stimulus for in vivo activation of these $\mathrm{T}$ cells, together with the induction of MS-like disease and CNS pathology. Ex vivo functional characteristics of $\mathrm{MOG}_{34-56}$ specific $\mathrm{T}$ cells are specific cytolysis of peptide
\end{abstract}

Electronic supplementary material The online version of this article (doi:10.1007/s11481-011-9320-5) contains supplementary material, which is available to authorized users.

S. A. Jagessar · N. Heijmans · B. A. 't Hart $(\square)$

Department of Immunobiology,

Biomedical Primate Research Centre,

PO Box 3306, 2280GH, Rijswijk, The Netherlands

e-mail: hart@bprc.nl

B. Gran $\cdot$ C. S. Constantinescu

Academic Division of Clinical Neurology,

University of Nottingham,

Nottingham, United Kingdom

J. Bauer

Department of Neuroimmunology, Medical University Vienna, Center for Brain Research,

Vienna, Austria

\section{J. D. Laman · B. A. 't Hart}

Department Immunology, Erasmus Medical Center,

Rotterdam, The Netherlands

S. A. Jagessar · J. D. Laman · B. A. 't Hart ErasMS Center for Translational research into MS, Rotterdam, The Netherlands pulsed target cells and high IL-17A production. To indentify possible functions in this new model of $\mathrm{T}$ helper 1 cells, which play a central pathogenic role in MS models induced with complete Freund's adjuvant, we tested the effect of human interferon- $\gamma$ (IFN $\gamma$ ) administration during disease initiation of the disease (day 0-25) and around the time of disease expression (psd 56-81). The results show a clear modulatory effect of early IFN $\gamma$ treatment on humoral and cellular autoimmune parameters, but no generalized mitigating effect on the disease course. These results argue against a prominent pathogenic role of $\mathrm{T}$ helper 1 cells in this new marmoset EAE model.

Keywords Common marmoset · T helper 1 IL-17 . Interferon-gamma $\cdot$ Multiple sclerosis .

Myelin/oligodendrocyte glycoprotein

\section{Introduction}

Experimental autoimmune encephalomyelitis (EAE) is a well-established animal model of the human central nervous system (CNS) targeting the neuroinflammatory disease multiple sclerosis (MS). Various versions of the model are being used to investigate immunopathogenic mechanisms in MS and to develop new therapies. In response to the need of an EAE model with high relevance to MS in which biological therapeutics can be tested, we have set up an EAE model in the Neotropical primate "common marmoset" (Callithrix jacchus). The model that was induced by sensitization against human myelin or the immunodominant component myelin/ oligodendrocyte glycoprotein (MOG) and its advantage 
over equivalent models in rodents has been discussed elsewhere, most recently in ('t Hart and Massacesi 2009).

Analogous to EAE models in lower species, such as mice and rats, $\mathrm{T}$ helper 1 (Th1) cells have a central pathogenic role in marmoset EAE models induced with recombinant human myelin/oligodendrocyte glycoprotein (rhMOG) in complete Freund's adjuvant (CFA) (Brok et al. 2000). Accordingly, antibodies against human CD40, a co-stimulatory molecule of antigen presenting cells involved in the induction of IL-12, or against IL-12p40 subunit displayed strong suppressive activity, both in a prophylactic (Boon et al. 2001; Brok et al. 2002; Laman et al. 2002) and in a therapeutic experimental setting ('t Hart et al. 2005a; b). However, the recent failure of the anti-IL12 p40 antibody (Ustekinumab) in a phase II clinical trial in relapsing-remitting MS points at an important discrepancy with MS (Segal et al. 2008). The question is therefore warranted whether the pathogenic role of Th1 cells in MS is comparable to that in EAE models.

We hypothesize that the dominant pathogenic role of Th1 cells in EAE models can be attributed to the use of CFA (Billiau and Matthys 2001). This would imply that EAE models induced without use of CFA are less sensitive to reagents that modulate Th1 functions. Interferon-gamma (IFN $\gamma$ ) is the principal Thl cytokine induced by IL-12 and has consistently been found to be protective in mouse models of EAE (Willenborg et al. 1999). In a mouse model, we showed that IL-12 suppressed disease when administered systemically during the early phase of EAE induction (Gran et al. 2004). The suppressive effect was dependent on IFN $\gamma$, as it was not observed in IFN $\gamma$-deficient mice. Moreover, genetic deficiency or antibody neutralization of IFN $\gamma$ consistently exacerbates EAE (Sanvito et al. 2010). On the other hand, in another mouse model, IL-12 given during the remission phase after the first attack induced more severe relapses, and restored EAE susceptibility in mice in which CD40-CD40 ligand interaction had been blocked. However, it is not known whether these effects are mediated by IFN $\gamma$ (Constantinescu et al. 1999).

The aim of the current study was to test the activity of human IFN $\gamma$ in a recently developed marmoset EAE model that is induced without usage of CFA, namely by immunization with a synthetic peptide representing the sequence 34 to 56 of human $\mathrm{MOG}\left(\mathrm{MOG}_{34-56}\right)$ formulated in incomplete Freund's adjuvant (IFA) (Jagessar et al. 2010). The development of characteristic MS-like clinical and pathological features in this model is driven by $\mathrm{CD}^{+}{ }^{+} \mathrm{CD} 4^{+} / \mathrm{CD} 8^{+} \mathrm{CD} 56^{+} \mathrm{CD} 16^{-} \mathrm{T}$ cells that display high IL-17A production and antigen-specific cytolysis as activities potentially contributing to EAE development (Jagessar et al. 2010). We have examined the effect of human IFN $\gamma$ administration during two periods, namely an early treatment period from the time of immunization to post sensitization day (psd) 25 and a late treatment period from psd 56 to 81 . The primary outcome measures were the susceptibility to, and the severity of clinical EAE. Secondary and exploratory outcome measures included immunological parameters and histological evidence of disease activity.

The data show a modulatory effect of early IFN $\gamma$ treatment on cellular as well as humoral autoimmune parameters, yet with only a marginal effect on the disease course. This finding argues against a generalised pathogenic role of the IL-12/IFN $\gamma$ axis in the marmoset EAE model induced with $\mathrm{MOG}_{34-56}$ /IFA.

\section{Materials and methods}

Animals

Fifteen healthy marmoset monkeys (Callithrix jacchus) were included in this study originated from purpose-bred colonies of the Biomedical Primate Research Centre (BPRC), Rijswijk, The Netherlands, and the German Primate Centre (DPZ), Göttingen, Germany. Monkeys purchased from DPZ had been housed for at least 6 months in the BPRC before use. Individual data of the monkeys used in this study are listed in Table 1. Before inclusion in the study, the monkeys were subjected to a complete physical, hematological and biochemical examination. During the study they remained under veterinary care. Monkeys were pair-housed in spacious cages enriched with branches and toys and with padded shelter provided on the floor. The daily diet consisted of commercial food pellets for New World monkeys (Special Diet Services, Witham, Essex, UK), supplemented with rice, raisins, peanuts, marshmallows, biscuits, fresh fruit, grasshoppers, and maggots. Drinking water was provided ad libitum.

Ethics

In accordance with the Netherland's law on animal experimentation, all study protocols and experimental procedures were reviewed and approved by the Institute's Ethics Committee before the start of experiments.

EAE induction

Synthetic MOG peptides based on the human MOG extracellular sequence, which were used for immunization and cell culture, were purchased from Cambridge Research Biochemicals (Cleveland, UK). 
Table 1 Overview of marmosets used in this study
${ }^{\mathrm{a}}$ Age in months at the start of the experiment; F, female; $\mathrm{M}$, male; psd, post sensitization day

Score $2.0=$ ataxia; score $2.25=$ incomplete paralysis of hind limbs

\begin{tabular}{|c|c|c|c|c|c|c|}
\hline Group & Name & Sex & $\operatorname{Age}^{a}$ & Sacrificed (psd) & Score 2.0 & Score 2.25 \\
\hline \multirow[t]{5}{*}{ Control } & M06054 & $\mathrm{F}$ & 32 & 99 & 56 & 95 \\
\hline & M07009 & M & 28 & 91 & 86 & 90 \\
\hline & M07034 & $\mathrm{F}$ & 25 & 113 & 93 & 101 \\
\hline & M07076 & $\mathrm{F}$ & 22 & 154 & $>154$ & $>154$ \\
\hline & Mi12699 & M & 59 & 133 & 100 & 108 \\
\hline Mean $\pm S D$ & & & & $118 \pm 26$ & $98 \pm 36$ & $110 \pm 26$ \\
\hline rhIFN- $\gamma$ & M04014 & $\mathrm{F}$ & 64 & 154 & $>154$ & $>154$ \\
\hline \multirow[t]{4}{*}{ Day0-25 } & M06036 & M & 35 & 133 & 127 & 128 \\
\hline & M07062 & $\mathrm{F}$ & 23 & 57 & 51 & 54 \\
\hline & M07101 & $\mathrm{F}$ & 20 & 135 & 119 & 120 \\
\hline & Mi13264 & M & 41 & 144 & 138 & 143 \\
\hline Mean $\pm S D$ & & & & $125 \pm 39$ & $118 \pm 40$ & $120 \pm 39$ \\
\hline rhIFN- $\gamma$ & M06063 & M & 32 & 127 & 100 & 110 \\
\hline \multirow[t]{4}{*}{ Day56-81 } & M07016 & $\mathrm{F}$ & 26 & 81 & 72 & 77 \\
\hline & M07035 & $\mathrm{F}$ & 25 & 155 & $>155$ & $>155$ \\
\hline & M07047 & $\mathrm{F}$ & 24 & 57 & 44 & 55 \\
\hline & Mi12700 & M & 59 & 155 & $>155$ & $>155$ \\
\hline Mean $\pm S D$ & & & & $115 \pm 44$ & $105 \pm 50$ & $110 \pm 45$ \\
\hline
\end{tabular}

The monkeys were sedated with Alfaxan $(10 \mathrm{mg} / \mathrm{kg})$ (Vétoquinol S.A., Magny-Vernois, France) and were immunized with an emulsion of $100 \mu \mathrm{g} \mathrm{MOG}_{34-56}$ dissolved in $300 \mu \mathrm{l}$ buffered saline with $300 \mu \mathrm{l}$ IFA (Difco Laboratories, Detroit, MI). The inoculum was injected into the inguinal and axillary regions of the dorsal skin divided over 4 spots of $150 \mu \mathrm{l}$ each. Antigen-adjuvant emulsions were prepared by gentle stirring the peptide/oil mixture at $4^{\circ} \mathrm{C}$ for at least $1 \mathrm{~h}$. Monkeys that failed to develop serious neurological deficit (EAE score $\geq 2.0$ ) were again immunized at psd 28 and 56 with the same dose of peptide in IFA.

\section{Treatment schedule}

Clinical grade recombinant human IFN $\gamma$ (Immukin $\left.{ }^{\circledR}\right)$ was purchased from Boehringer Ingelheim, Vienna, Austria. Biological activity in the marmoset system was confirmed by increase of MHC class II expression on marmoset PBMC (Suppl. Fig. 1).

Three groups of 5 animals each were randomly created and as outlined in Fig. 1. Power calculation was used to calculate the minimal group size for statistical evaluation (Mann-Whitney $U$ test). Based on historical data we assumed a $100 \%$ disease incidence. To achieve a statistical power of $80 \%$ the group size should be 5 . The control group received 3 subcutaneous injections per week (Mon, Wed, Fri) of buffered saline $(1.0 \mathrm{ml} / \mathrm{kg})$ during two treatment episodes from psd $0-25$ and psd 56-81. The early IFN $\gamma$ treatment/prophylactic group) and the late IFN $\gamma$ treatment/therapeutic group) received 3 subcutaneous test substance injections at a dose of $1.5 \mu \mathrm{g} / \mathrm{kg}(1.0 \mathrm{ml} / \mathrm{kg})$ per week during psd 0-25 and psd 56-81, respectively.

\section{Clinical scoring}

Clinical signs were scored daily by two independent observers as described ("t Hart et al. 1998). Briefly: $0=$ no clinical signs; $0.5=$ apathy, loss of appetite, altered walking pattern without ataxia; 1=lethargy, anorexia,

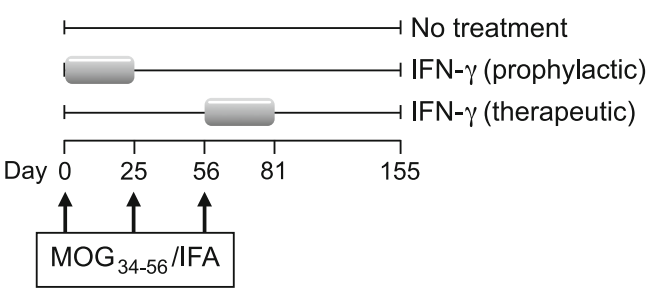

Fig. 1 Design of experiment. Fifteen marmosets of either sex were randomly divided over 3 groups, 5 animals in each group (see table 1). All marmosets were immunized with $\mathrm{MOG}_{34-56}$ in IFA and were rechallenged at days 25 and 56 . The first group (control) received only solvent of the drug recombinant human IFN $\gamma$ between post sensitization day (psd) $0-25$ and psd 56-81. The second group (prophylactic treatment) received three subcutaneous injections of human IFN $\gamma$ $(1.5 \mu \mathrm{g} / \mathrm{kg})$ per week (Mon-Wed-Fri) between psd $0-25$; the third group (therapeutic treatment) received the same injection schedule between psd $56-81$ 
loss of tail tonus, tremor; $2=$ ataxia, optic disease; $2.5=$ para- or monoparesis, sensory loss, brain stem syndrome; 3 =para- or hemiplegia; $4=$ quadriplegia; $5=$ spontaneous death attributable to EAE. Monkeys were sacrificed for ethical reasons once complete paralysis of one or both hind limbs (score $\geq 3.0$ ) was observed, or at a pre-determined endpoint.

Body weight, measured three times per week, served as an objective surrogate disease marker. Weighing was performed without sedation using the perspex cylinder: adequately trained monkeys were captured from the home cage.

\section{Necropsy}

Monkeys selected for necropsy were first deeply sedated by intramuscular injection of alfaxan $(10 \mathrm{mg} / \mathrm{kg})$. Maximum blood volume was collected into heparinized vacutainers and subsequently the marmoset was euthanized by infusion of pentobarbital sodium (Euthesate ${ }^{\circledR}$; Apharmo, Duiven, The Netherlands).

Spleen and several lymph nodes were collected aseptically and cut into four pieces, which were used for cell culture or stored in $4 \%$ formalin, $-80^{\circ} \mathrm{C}$, and RNAlater (Sigma, St. Louis, MO). Half of the brain and spinal cord were stored in $4 \%$ formalin and the other half were snapfrozen in liquid nitrogen.

\section{Cell preparation}

The maximum monthly blood volume that can be collected from marmosets is $1 \%$ of the body weight, which is $3.5-$ $4.0 \mathrm{ml}$ for an average adult monkey of 350-400 g. Longitudinal immune monitoring was performed using $1.5 \mathrm{ml}$ venous blood collected at 2 weeks interval from the femoral vein into heparinized vacutainers (Greiner, Sölingen, Germany).

At necropsy mononuclear cell (MNC) suspensions were prepared from aseptically removed spleen and axillary (ALN), inguinal (ILN), cervical (CLN) and lumbar (LLN) lymph nodes. MNC and PBMC were isolated using lymphocyte separation medium (LSM ${ }^{\circledR}$, ICN Biomedical Inc., Aurora, $\mathrm{OH})$.

\section{Proliferation of $\mathrm{T}$ cells}

PBMC and lymphoid organ MNC suspensions were assayed in triplicate for proliferation against rhMOG $(10 \mu \mathrm{g} / \mathrm{ml})$ and a panel of MOG peptides (each $10 \mu \mathrm{g} / \mathrm{ml}$ ) (Jagessar et al. 2010). Proliferation was assessed by the incorporation of $\left[{ }^{3} \mathrm{H}\right]-$ thymidine $(0.5 \mu \mathrm{Ci} /$ well $)$ during the final $18 \mathrm{~h}$ of $64 \mathrm{~h}$ culture using a matrix $9600 \beta$-counter (Packard 9600; Packard Instrument Company, Meriden, CT). Results are expressed as stimulation index (SI), being the ratio of radiolabel incorporation in stimulated versus unstimulated cultures. SI values above 2.0 were considered positive.

Flow cytometry

Cells were phenotyped as described previously (Jagessar et al. 2010). Flow cytometric analysis was performed on a FACS LSRII flow cytometer using FACSDiva software 5.0 (BD Biosciences).

\section{Cytokines}

Supernatants of PBMC, ALN, and spleen MNC were collected after $48 \mathrm{~h}$ stimulation with rhMOG or a panel of overlapping MOG peptides. Supernatants were assayed according to manufacturers instructions with commercial ELISA kits for monkey IL-10, monkey IFN $\gamma$ (U-Cytech, Utrecht, The Netherlands) and human IL-17A (eBioscience, San Diego, CA).

\section{Quantitative PCR}

Total RNA was extracted from PBMC, spleen and ALN using RNeasy minikit (Qiagen, Hilden, Germany) and subsequently cDNA was synthesized using RevertAid First Strand cDNA Synthesis Kit (Fermentas, St. Leon-Rot, Germany) according to the manufacturer's instructions. Random hexamer primers were used for cDNA synthesis. Quantitative PCR was performed in duplicate using a using iTaq supermix with ROX and CFX96 Real-Time System (both from Bio-Rad, Hercules, CA). The primers (Invitrogen) and probes (purchased from the Universal Probe Library set for human, Roche, Indiapolis, In) used are listed in Table 2. mRNA expression of tested primers was related to mRNA expression of the reference gene ABL $\left(2^{\mathrm{Ct} \text { reference }-\mathrm{Ct} \text { target }}\right)$.

\section{Autoantibody detection}

Plasma samples were analyzed for $\operatorname{IgG}$ and $\operatorname{IgM}$ antibody binding to rhMOG or to a panel of overlapping 23-mer pMOG sequences using ELISA as described (Boon et al. 2001); (Jagessar et al. 2008). Bound $\operatorname{IgG}$ and $\operatorname{IgM}$ were detected using polyclonal alkaline phosphatase-conjugated rabbit-anti-human IgG (Abcam, Cambridge, UK) or goat-anti-monkey IgM (Rockland, Gilbertsville, PA). Antibody binding was measured at $405 \mathrm{~nm}$ and results are expressed in arbitrary units (AU) using the software ADAMSEL (developed by Dr. E. Remarque, Biomedical Primate Research Centre, Rijswijk, The Netherlands). 
Table 2 Primers with corresponding probes used for quantitative PCR

\begin{tabular}{llll}
\hline Gene & Forward primer & Reverse primer & Probe \\
\hline ABL & CAGAGAAGGTCTATGAACTCATGC & GGTGGATTTCAGCAAAGGAG & 86 \\
IL-1 $\beta$ & TGGTCCTAAACAGATGAAGTGC & GTAGTGCTGGCGGGAGAGT & 85 \\
IL-2 & AAGTTTTACATGCCCAAGAAGG & AAGTGAAAGTTTTGCTTTGAGCTA & 65 \\
IL-10 & GTTGCCTTCAGCAGAGTGAA & GCAACCCAGGTAACCCTTAAA & 67 \\
IL-17A & CCTCATTGGTGTCACTGCTG & TGCAATTCCTGCCTTCACTA & 146 \\
IFN $\gamma$ & GGAGAGAGGAGGGTGACAGA & TTGGATGCTCTGGTTGTCTTTA & 21 \\
TNF $\alpha$ & GGACGAGCTCTCCAAGGACT & GTCACTCGGGATTCGAGAAG & 81 \\
CD3 & AGGCAAGAGTGTGTGAGAACTG & GATGCAGATGTCCACTATGACAA & 10 \\
CD4 & TCTGTGAAGTGGAGAGCAAAAA & TGACAGTCAATCCGAACACC & 10 \\
CD8 & TCATGTACTTCAGCAGCTTCG & GCTCTGGTGTGGGTGGTC & 4 \\
\hline
\end{tabular}

A
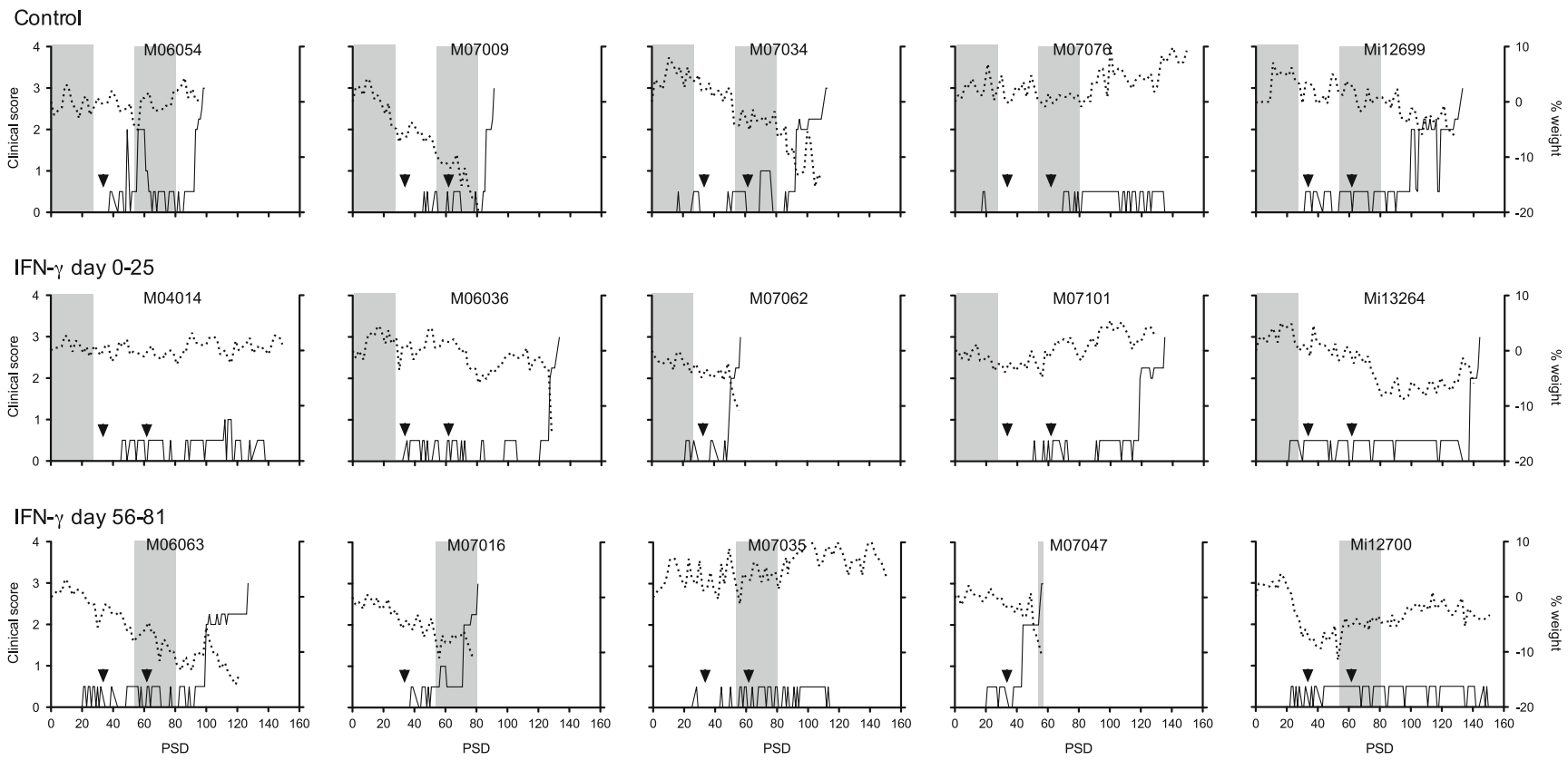

B
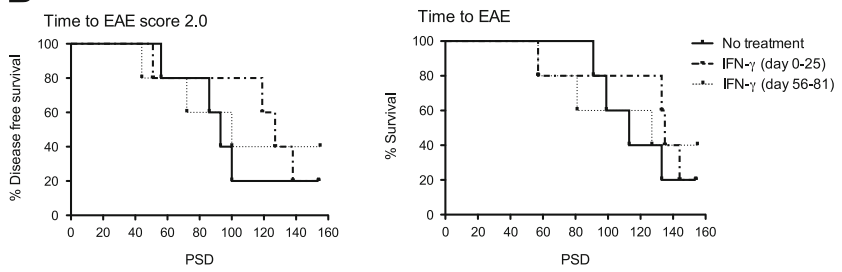

Fig. 2 Clinical scores and body weights of all animals. Marmoset monkeys $(n=15)$ were immunized with $\mathrm{MOG}_{34-56}$ in IFA and subsequently treated for a period of 25 days with recombinant human interferon-gamma by intravenous injection at a frequency of 3 times per week (see Fig.1). a The period of IFN- $\gamma$ treatment is shown as grey shaded areas. The control group received only the solvent of IFN- $\gamma$. Left $y$-axis (solid line) indicates clinical scores and right $\mathrm{y}$-axis (dotted line) the percentage body weight loss relative to

day 0 as a surrogate disease marker. On the $\mathrm{x}$-axis time after immunization is plotted as post sensitization day. Arrowheads indicate day of booster-immunizations with $\mathrm{MOG}_{34-56}$ in IFA. M07062, M06054 and M07016 were boosted only once. PSD; post sensitization day. b Survival curves are shown where the control group is compared to the IFN- $\gamma$ treatment groups. In the left panel time to EAE score 2.0 and in the right panel time to the ethical endpoint (EAE score 3.0) is depicted 


\section{Histology}

Formalin-fixed brain sections were used for histological examination as described previously ("t Hart et al. 1998; Jagessar et al. 2008; 2010). For quantification of demyelination 8 brain sections, in total $6 \mathrm{~cm}^{2}$, were stained with Luxol fast blue - periodic acid Schiff (LFB-PAS). The Scion Image Program (NIH, USA) was used to calculate the amount of demyelination per $\mathrm{mm}^{2}$ as total of the white and grey matter. Another set of 8 brain sections was stained with hematoxylin and eosin and CD3 to determine the amount of inflammation.

\section{Statistical analysis}

A high variation of the disease course and associated immune parameters between individual animals is inherent to the outbred nature of this model. Where possible data were
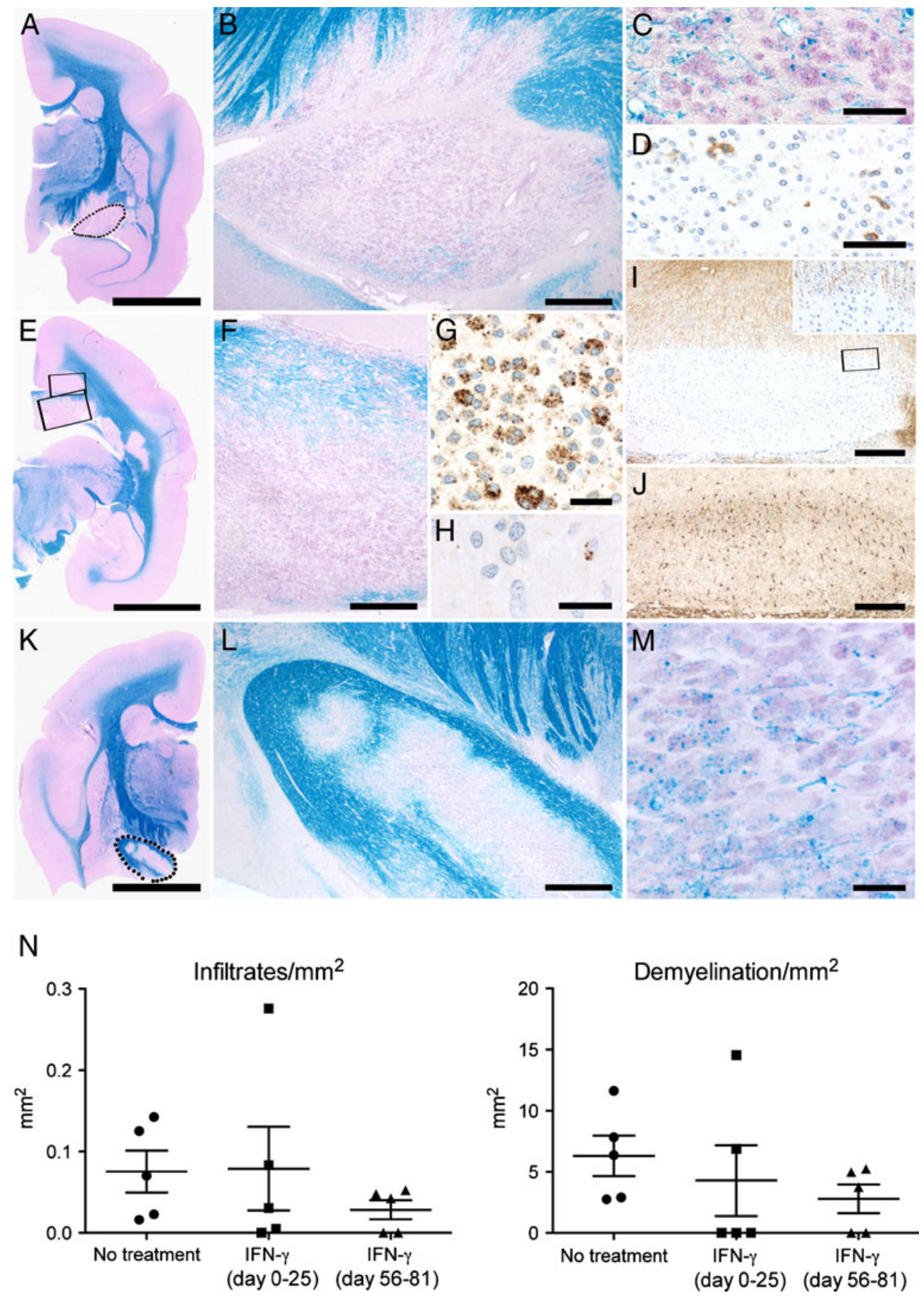
analyzed using the Mann-Whitney $U$ test; $p$ values $<0.05$ are considered to be significant.

\section{Results}

Human IFN $\gamma$ has a variable effect on the disease course in $\mathrm{MOG}_{34-45}$ induced EAE

Fifteen unrelated marmosets from an outbred colony were randomized over three experimental groups of 5 monkeys each (see Table 1), the group size was determined by power calculation (see Materials and Methods) (Bacchetti et al. 2011). EAE was induced by immunization with human $\mathrm{MOG}_{34-56}$ in IFA. Despite the absence of ligands of innate receptors for antigen presenting cell activation this procedure has induce clinically evident EAE in almost all tested monkeys. Sometimes a case is found without evident neurological signs within the predetermined observation period, but such cases do display the characteristic CNS pathology. Human IFN $\gamma$ was administered during 25 days between the first and second immunization (psd 0-25), modeling a prophylactic treatment regimen, or for 25 days after psd 56 (psd 56-81), modeling therapeutic treatment (Fig. 1).

4 Fig. 3 Inflammation and demyelination in the brain characterized by histology and immunohistochemisty. a-j Brain of M07034 as a representative of the control group. a A low magnification scan from a Kluver-Barrera (KLB) stained section (bar: $5 \mathrm{~mm}$ ). The encircled area represents the demyelinated optic tract, which is depicted enlarged in b (bar: $500 \mu \mathrm{m}$ ). c Luxol fast blue - periodic acid Schiff (LFB-PAS) staining shows in the optic tract the presence of macrophages with intracellular myelin degradation products (bar: $50 \mu \mathrm{m})$. d Immunostaining for $\mathrm{CD} 3$ reveals the presence of some single $\mathrm{T}$ cells in the optic tract (bar: $50 \mu \mathrm{m}$ ). e Another brain slice of M07034 stained for KLB (bar: $5 \mathrm{~mm}$ ) is shown. The lower rectangle shows demyelination in the corpus callosum that is enlarged in $\mathbf{f}$ and contains LFB-PAS positive macrophages (bar: $500 \mu \mathrm{m}$ ). $\mathbf{g}$ Immunostaining for myelin proteolipid protein (PLP) shows macrophages containing PLP degraded products (bar: $25 \mu \mathrm{m}$ ). h A single Granzyme B positive cytotoxic $\mathrm{T}$ cell is shown in the corpus callosum (bar: $20 \mu \mathrm{m}$ ). The upper rectangle in $\mathbf{e}$ shows a cortical area that is shown enlarged in $\mathbf{i}$ (bar: $250 \mu \mathrm{m}$ ) and in a PLP staining reveals subpial demyelination. The rectangle in I shows the edge of the lesion, which is enlarged in the insert. $\mathbf{j}$ Although no PLP positive degradation products are present, staining for macrophage/microglia marker Iba-1 identifies increase of microglial cells at the border of the subpial lesion (bar: $250 \mu \mathrm{m})$. $\mathbf{k}$ A low magnification scan of a KLB stained section from M07035 as representative of the late IFN $\gamma$ treated group. The encircled area again shows the optic tract, which is only partially demyelinated and enlarged in $\mathbf{I}$ (bar: $500 \mu \mathrm{m})$. $\mathbf{m}$ Further enlargement of $\mathrm{L}$ reveals the presence of macrophages with myelin degraded products (bar: $25 \mu \mathrm{m}$ ). n For each animal in the three groups 8 brain sections were analysed, which is comparable with $6 \mathrm{~cm}^{2}$ in total. The amount of infiltrated cells per $\mathrm{mm}^{2}$ in the brain is given. Same quantification is done for the amount demyelination in the brain. A detailed description of the calculation is given in 'Material and Methods'
The results are expressed as clinical course of individual animals, demonstrating that in each group 1 monkey did not display evident neurological deficit (Fig. 2). Besides, a second monkey in the therapeutic group (Mi12700) did not show neurological symptoms but suffered from weight loss. The actual survival times of individual animals depicted in table 1 show that the time interval to the first symptom of overt neurological disease (ataxia; score 2.0) was delayed from 98 to 118 days by early treatment with IFN $\gamma$ and from 98 to 105 days in monkeys receiving late treatment with IFN $\gamma$. Two parameters were statistically analyzed, namely disease-free survival (time to EAE score 2.0) and overall survival (time to the ethical end-point: score 3.0) (Fig. 2b). The survival curves show that neither the prophylactic, nor the therapeutic treatment with IFN $\gamma$ had a consistent positive effect on the disease course.

\section{Demyelination and inflammation in brain}

Pathological differences between the three groups were examined by histology and immunohistochemistry. As representative example of the control group and IFN $\gamma$ treated group, respectively, M07034 and M07035 are shown in Fig. 3. Brain sections in both animals showed demyelinated areas populated by activated macrophages that contain degraded myelin products and by infiltrated $\mathrm{CD}^{+} \mathrm{T}$ cells. Demyelinated lesions were also observed in the optic tract and in the corpus callosum. Interestingly, Granzyme B staining detected some cytotoxic $\mathrm{T}$ cells. Quantification of the amount of inflammation and demyelination in brain for each animal (Fig. 3n), revealed no differences in the intensity of demyelination between the control and IFN $\gamma$ treated groups. However, inflammation tended to be less intensive in the late treatment group (psd 56-81) although differences were not statistically significant.

\section{Modulation of humoral immunity by IFN $\gamma$}

Immunization with $\mathrm{MOG}_{34-56}$ in CFA or IFA induces IgM and IgG antibodies binding to ELISA-plate bound overlapping peptides, i.e. $\mathrm{MOG}_{24-46}$ and $\mathrm{MOG}_{34-56}$, but not to rhMOG protein (Jagessar et al. 2008), (2010). Results of the analysis of serial immune sera from the current experiment are depicted in supplementary Fig. 2. The data from individual monkeys and the mean areas under the curve (AUC) show that the highest IgM antibody levels against both peptides were measured in the early (psd 0-25) IFN $\gamma$ treated animals, although differences between the groups were not statistically significant. Figure $4 \mathrm{a}$ shows that $\operatorname{IgG}$ antibody levels against both peptides were significantly reduced in early 


\section{A}

MOG24-46
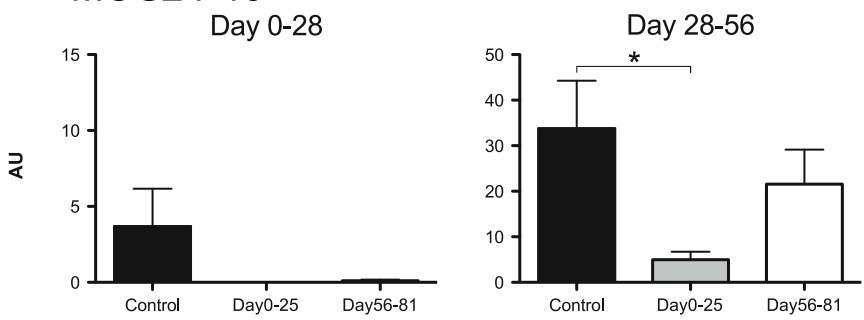

MOG34-56
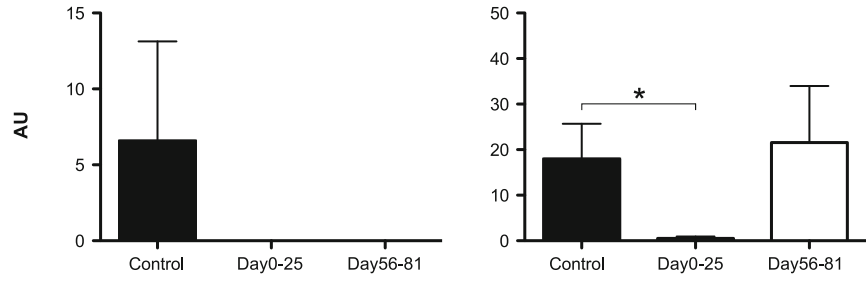

B

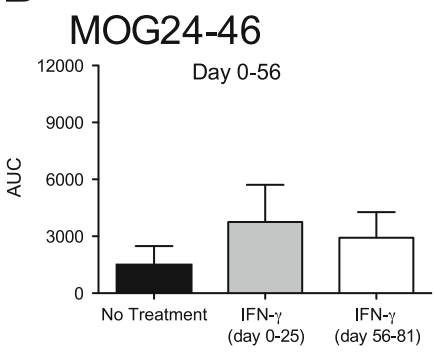

\section{MOG34-56}

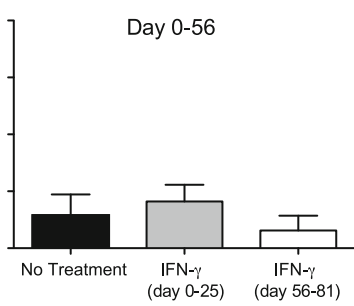

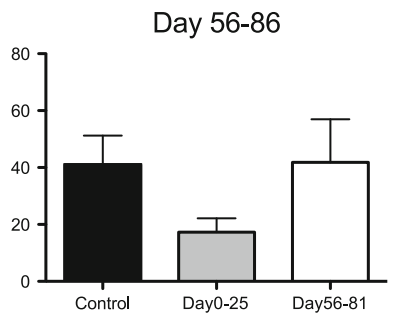
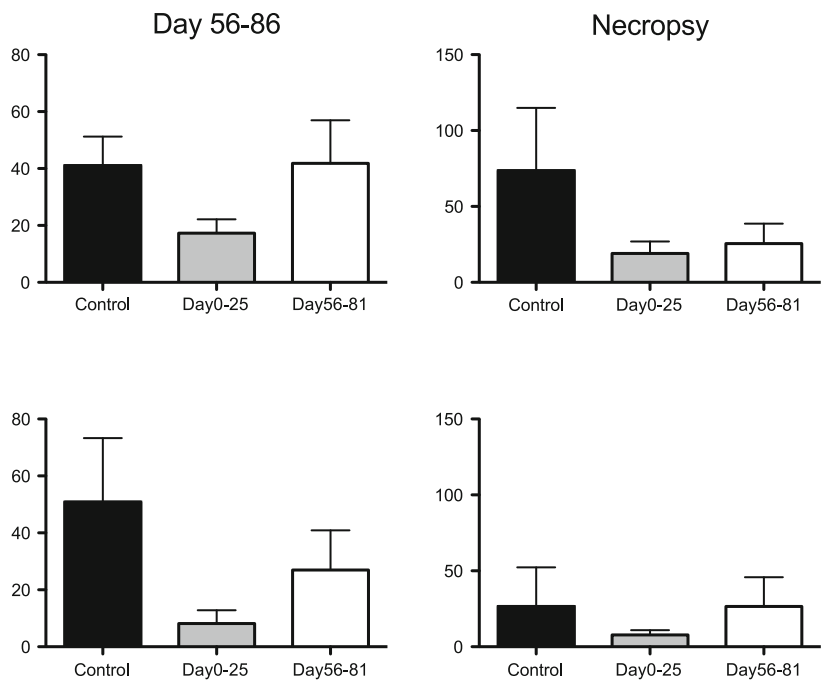

Fig. 4 Plasma $\operatorname{IgG}$ and $\operatorname{IgM}$ antibody binding to MOG peptides. Longitudinally collected plasma samples ( 2 weeks interval and at necropsy) were tested for the presence of $\operatorname{IgG}$ and $\operatorname{IgM}$ binding to ELISA plates coated with a panel of overlapping MOG peptides. Only antibodies binding to $\mathrm{MOG}_{24-46}$ and $\mathrm{MOG}_{34-56}$ were detected. a IgG plasma levels were compared during and after the treatment periods to assess whether there is a significant difference in antigen binding. Data are processed with ADAMSEL and expressed in arbitrary units (AU). b IgM antibody responses were only compared from day 0-56. Data are expressed in area under curve (AUC), which is calculated from data of Suppl. Fig. 2. ${ }^{*} \mathrm{p}<0.05$, Mann-Whitney $U$ test was used as statistical calculation. Data are presented as mean \pm SEM treated monkeys. Bar diagrams show mean antibody concentrations, which were calculated for the four time blocks, i.e. the no-treatment interval (psd 28-56). Summarizing, early treatment with IFN $\gamma$ resulted in suppressed IgG antibody production, whereas IgM antibody levels were not affected by the treatment.

\section{Modulation of cellular immunity by $\mathrm{IFN} \gamma$}

The modulation of cellular immunity in the model was examined with two ex vivo assays, namely antigen-induced proliferation and cytokine induction.

Proliferation of PBMC during the EAE course Every 14 days PBMC were collected and tested for proliferation against $\mathrm{MOG}_{34-56}$ or rhMOG. As shown in Fig. 5a responses against $\mathrm{MOG}_{34-56}$ were much stronger than against rhMOG and the highest responses were measured in the control monkeys from group 1. SI values $>2.0$ were regarded as positive. Interestingly, Fig. 5b shows that early treatment with IFN $\gamma$ significantly prolonged the time to reach SI values of 3.0, whereas this was not observed for the late treatment group.

Proliferation of PBMC and lymphoid organ MNC at necropsy Our previous study demonstrated that only a minor quantity of the $\mathrm{T}$ cells proliferating against anti$\mathrm{MOG}_{34-56}$ is present in blood, and that the vast majority can be found in the lymphoid organs. Hence we also tested the antigen reactivity of several secondary lymphoid organs collected at necropsy, namely spleen, the ALN and ILN that drain the immunization sites, and the LLN and CLN that respectively drain the spinal cord and brain.

It is of note that the proliferation data at necropsy did not provide an explanation for the absence of 
A
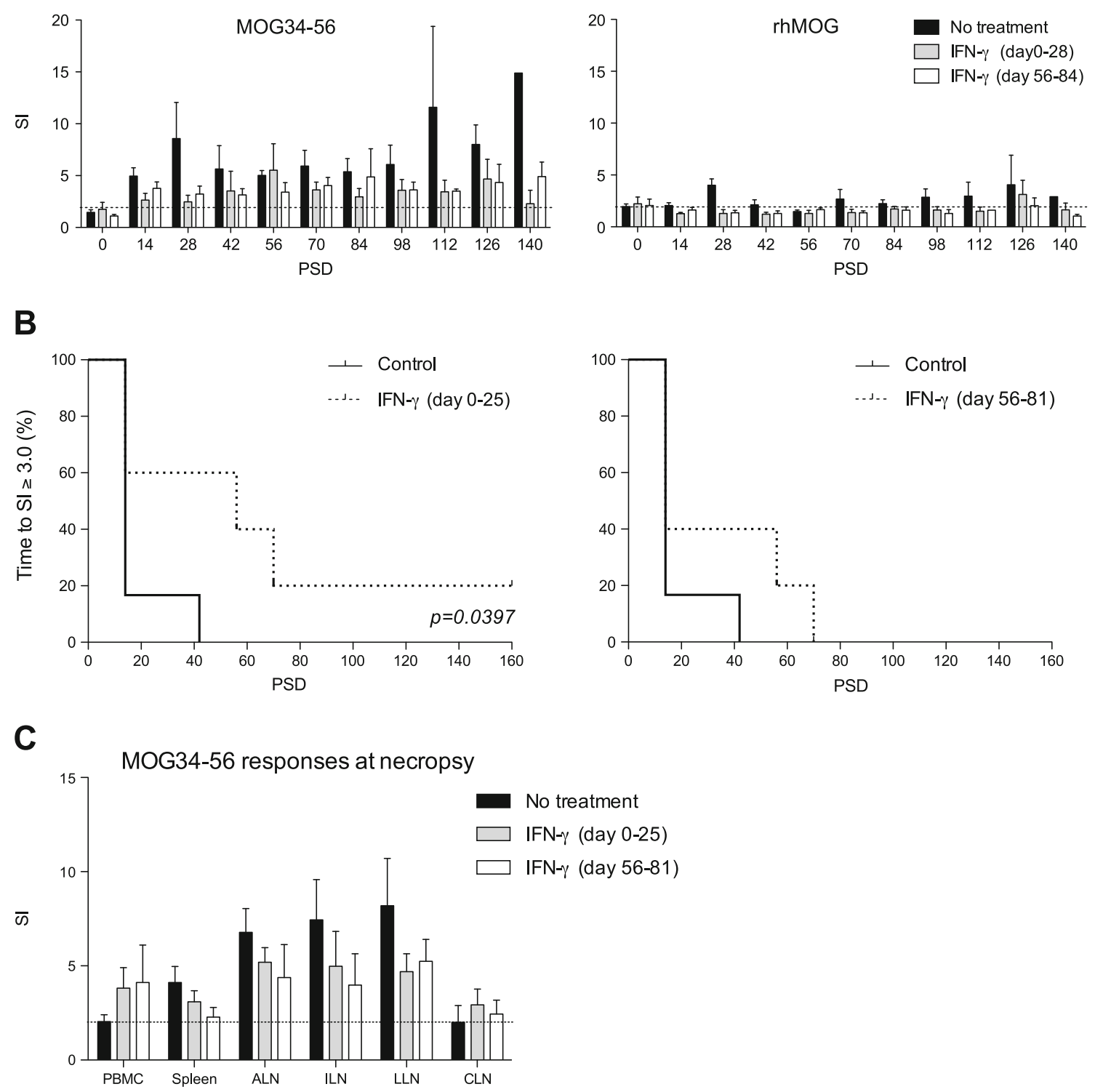

Fig. 5 T-cell proliferation against $\mathrm{MOG}_{34-56}$ and rhMOG. Mononuclear cells (MNC) isolated from venous blood (PBMC) or various lymphoid organs were probed for their proliferative response against $\mathrm{MOG}_{34-56}$ and rhMOG. Proliferation was quantified as the incorporation of $\left[{ }^{3} \mathrm{H}\right]$-thymidine in the final $18 \mathrm{~h}$ of 3 days culture. All data are expressed as stimulation index (SI) relative to unstimulated cultures. SI above 2 (dotted line) is considered positive. a Longitudinal reactivity of $\mathrm{PBMC}$ to $\mathrm{MOG}_{34-56}$ (left panel) and rhMOG (right

neurological signs in the EAE affected control monkey M07076. This monkey's proliferation profile was similar to that of control monkey Mi12699, which did develop clinical EAE. By contrast, the proliferation was markedly suppressed in monkeys from both IFN $\gamma$-treated groups that did not develop overt neurological disease, i.e. M04014 and M07035. Overall the results show that the proliferation profiles of individual monkeys differ markedly within each group (data not shown), but that panel) is shown. b Time to a stimulation index (SI) of 3.0 against $\mathrm{MOG}_{34-56}$ in blood was compared between the control group and IFN$\gamma$ treated groups. Log-rank was used to calculate $p$-value. c At necropsy MNC were prepared from blood, spleen, axillary (ALN), inguinal (ILN), lumbar (LLN), and cervical (CLN) lymph nodes. Only proliferation data from cultures stimulated with $\mathrm{MOG}_{34-56}$ are shown. Mann-Whitney $U$ test was used as statistical calculation. Data are presented as mean $\pm \mathrm{SEM}$

there are no obvious differences between the three groups (Fig. 5c). These data suggest that IFN $\gamma$ treatment did not exert a consistent positive or negative effect on $\mathrm{T}$ cell responses.

\section{Cytokines}

The main induced cytokine by ex vivo stimulation of PBMC with $\mathrm{MOG}_{34-56}$ or rhMOG was IL-17A, as 
measured by ELISA based on cross-reactive reagents. This was confirmed in the current study (Suppl. Fig. 3). Expression profiles of IL-17A, IFN $\gamma$ or IL-10 did not differ significantly between the three groups, although IL17A production after the first (psd 0) and third immunization (psd 56) was consistently observed in the monkeys that received IFN $\gamma$ treatment. To investigate this further we performed qPCR analysis of mRNA transcripts extracted from PBMC, spleen and ALN for a broader range of cytokines (Fig. 6a). The qPCR analysis showed no differences in cytokine expression between IFN $\gamma$-treated groups and the control group. However, transcript levels differed statistically between the organs (PBMC, spleen and ALN), mainly for IL-10, IL-1 $\beta$, TNF $\alpha$ and IL-2. A higher expression of IL-10 and TNF $\alpha$ was detected in spleen and ALN compared to PBMC in all three groups. The opposite effect was observed for IL-1 $\beta$, i.e. a higher expression in PBMC than in ALN or spleen. Level of expression of IL-2 was only higher in ALN compared to PBMC and spleen. Differences for the other two tested cytokines, IFN $\gamma$ and IL-17A, were less explicit.

\section{Discussion}

The optimal immunotherapy for MS selectively targets only the immune processes that cause the progressive accumulation of neurological deficit, while sparing immune functions protecting against infections or tumorigenesis (Feldmann and Steinman 2005). In the past years we have dissected the core pathogenic autoimmune factor against human CNS myelin in a unique EAE model in marmosets. The well-documented clinical and pathological similarity with MS is particularly relevant for translational research into pathogenic mechanisms as well as for therapy development ('t Hart et al. 2004; 2006).

The original model was induced by immunization with CNS myelin from human MS patient emulsified in CFA ('t Hart et al. 1998). The discovery that autoimmunity against MOG is essential for the induction of clinically evident EAE ('t Hart et al. 1998) led us to develop a reproducible disease model induced with rhMOG protein in CFA. Subsequent fine-specificity analysis revealed that the progression rate to full-blown clinical disease was associated with the activation of $\mathrm{CD}^{+} \mathrm{CD}^{+} / 8^{+} \mathrm{CD} 56^{+}$ cytotoxic T cells specific for $\mathrm{MOG}_{34-56}$ (Kap et al. 2008). The strong clinical effect of antibodies against human CD40 and against the shared p40 subunit of IL-12 and IL-23 underlined the important role of the CD40/IL-12/23 axis in the rhMOG-induced EAE model in marmosets (Boon et al. 2001; 't Hart et al. 2005a, b). Up to that point, the marmoset EAE model reproduced the essential pathogenic features of rodent EAE models.
However, further research led to the unexpected finding that immunization with $\mathrm{MOG}_{34-56}$ in IFA could also induce full-blown clinical EAE (Jagessar et al. 2010). The immunopathogenic mechanism of this new model was found to be driven by $\mathrm{T}$ cells that are activated without involvement of the otherwise required Toll-like receptor ligands and which induce widespread CNS demyelination in the absence of antibodies binding MOG protein. Fine-specificity analysis of this model revealed a central role of $\mathrm{T}$ cells specific for MOG peptide 40-48 with a remarkable phenotype, namely $\mathrm{CD}^{+} \mathrm{CD} 4^{+} \mathrm{CD} 8^{+} \mathrm{CD} 16^{-} \mathrm{CD} 27^{+} \mathrm{CD} 28^{-} \mathrm{CD} 45 \mathrm{RO}^{-}$. The $\mathrm{MOG}_{34-56}$ specific $\mathrm{T}$ cell lines generated from this model shows two immunological hallmarks i.e. high IL-17A production and specific cytolytic activity towards peptide presented via Caja-E molecules expressed by EBVtransformed autologous B-lymphoblastoid cells (Jagessar et al. 2011, in press).

These features clearly distinguish the new model from the MHC class II-restricted Th1 dominated classical EAE models in rodents and marmosets induced with CFA. To analyze this in further detail we have tested to what extent modulation of Th1-mediated pathogenic mechanisms by treatment with human IFN $\gamma$ modulates the disease course in this new model. Our prior expectation was analogous to the situation in mouse models of EAE (Sanvito et al. 2010) or of collagen-induced arthritis (Mauritz et al. 1988), presence of high IFN $\gamma$ levels during disease induction stimulated the disease, whereas late administration is suppressive. An explanation for the early stimulation is a generalized immune stimulatory effect, whereas the late suppressive effect might be that injection of IFN $\gamma$ suppresses production of IFN $\gamma$ at the tissue level (Brok et al. 1993).

In the marmoset EAE model, administration of IFN $\gamma$ between psd 0 and 25 exerted modulatory effects on several immunological parameters demonstrating in vivo biological activity, but had no systemic effect on the time of onset or the clinical course of the EAE model. Importantly, different from the observation in MS patients (Panitch et al. 1987), administration of IFN $\gamma$ did not aggravate clinical signs.

The body weight loss is a useful objective disease parameter, which is usually attributed to a systemic metabolic effect of inflammatory mediators such as TNF $\alpha$, IL-6 or IFN $\gamma$, the cachectic syndrome (Cahlin et al. 2000). The body weight loss in the $\mathrm{MOG}_{34-56}$ /IFA model is less serious than in the EAE models induced with CFA and shows a high interindividual variability that is inherent to the outbred nature of the marmoset EAE model. Mean body weight measurements of the early IFN $\gamma$-treated animals remained stable between psd 0 and 56, whereas a decrease was observed in the control and late treated animals, which 
Fig. 6 Cytokine expression measured by qPCR. mRNA was extracted from PBMC, spleen and ALN that were aseptically collected at necropsy and from PBMC collected at 2 weeks interval during the disease course. Primers with the corresponding probes as detailed in Table 2 were used for qPCR. a IFN $\gamma$, IL-17, IL10, IL-1 $\beta$, $\mathrm{TNF} \alpha$ and IL-2 mRNA level in PBMC, spleen and ALN were determined in all three groups. Data depicted in the graph were expressed relative to the household gene ABL (see Materials \& Methods). b CD3 expression was also determined in PBMC, spleen and ALN. MannWhitney $U$ test: ${ }^{*} p<0.05$; $* * p<0.001$
A
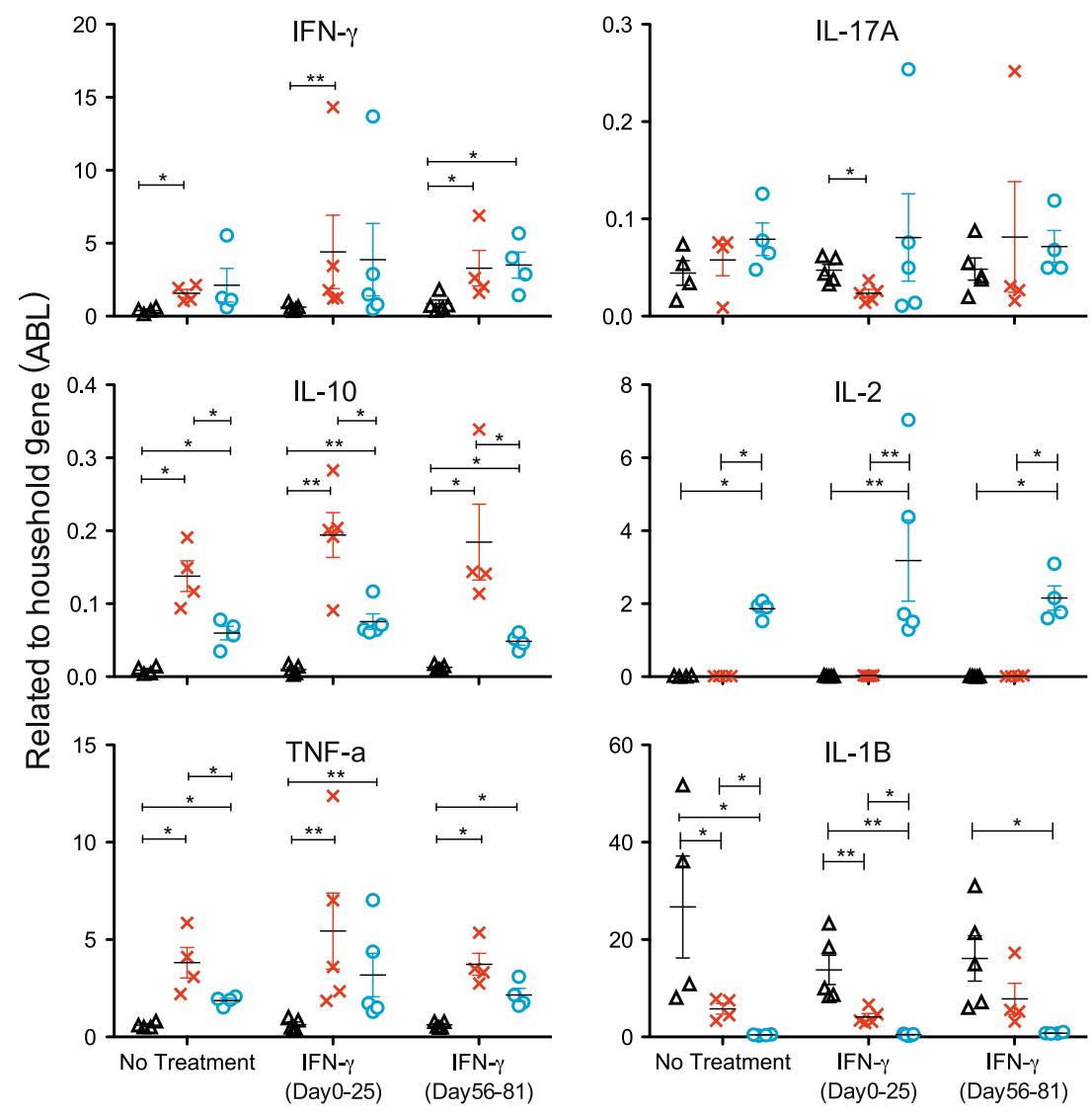

$\triangle$ PBMC $\times$ Spleen $\circ$ ALN

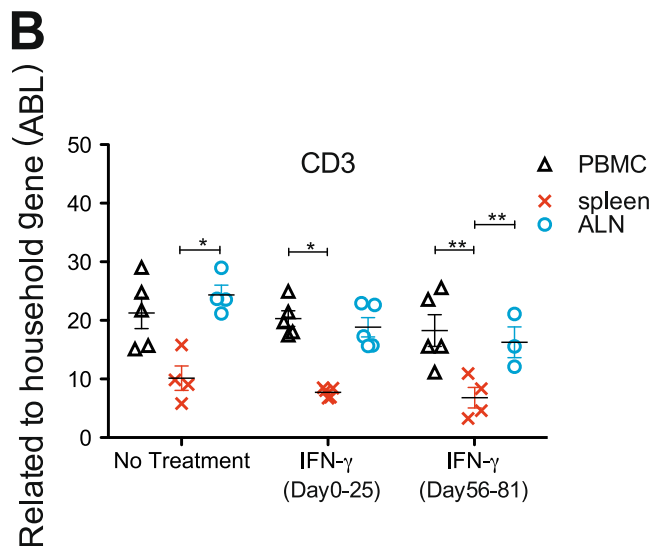

were untreated during this time period. Late treatment with IFN $\gamma$ during the episode psd 56 to 81 had no clear effect on the mean weight loss. It is tempting to speculate that the variable clinical effect of IFN $\gamma$ may be due to a similar variation in immunopathogenic profile as observed in MS patients (Axtell et al. 2010).

Another level of modulation by IFN $\gamma$ was the skewing of the IgM or IgG ratio of anti-MOG peptide antibodies, i.e. the increase of $\operatorname{IgM}$ and decrease of $\operatorname{IgG}$.
This was less evident in the late treatment group. Although proliferation of PBMC remained relatively low, it was affected by the IFN $\gamma$ treatment. The time to exceed the SI threshold 3 was delayed in the early treatment group. Moreover, PBMC from IFN $\gamma$ treated monkeys proliferated less against $\mathrm{MOG}_{34-56}$ and rhMOG than PBMC from control monkeys during late stage disease, i.e. after psd 100 . However, the reactivity profiles of MNC from the lymphoid organs were largely compa- 
rable between the three groups. At the level of cytokine production no effect of IFN $\gamma$ treatment was observed, only the lymphocytes from the various organs within each group showed significant differences. Differences between PBMC, spleen and ALN were mainly found in the expression of IL-10, IL-1 $\beta$, TNF $\alpha$ and IL-2.

Treatment with human IFN $\gamma$ exerts several modulatory effects on immune parameters in the new EAE model, in particular when the cytokine is administered during the induction of the pathogenic process. These effects are more subtle than those observed in regular EAE models induced with CFA. Despite these immunomodulatory effects, IFN $\gamma$ treatment had no clear effect on the severity nor the course of neurological disease. We would like to propose as explanation that the IL-17A producing cytotoxic $\mathrm{CD}^{+} \mathrm{CD}^{+} / \mathrm{CD} 8^{+} \mathrm{CD} 56^{+} \mathrm{CD} 16^{-} \mathrm{T}$ cells that form the core pathogenic factor in the $\mathrm{MOG}_{34-56} / \mathrm{IFA}$ EAE model are not affected by IFN $\gamma$ treatment.

In conclusion, human IFN $\gamma$ is biologically active on several immune parameters in the marmoset EAE model. Nevertheless, we observed neither of the early nor the late treatment a detectable effect on the severity or course of EAE. This marks a clear difference with rodent EAE models. We also did not observe aggravation of clinical signs as was observed in relapsing remitting MS patients. Our interpretation of this discrepancy is that the T cells that cause pathology and neurological deficit in this model are committed effector memory cells (Jagessar et al. 2011, in press), which exert their activity in the late progressive phase of the disease (Kap et al. 2008). Taken together the conclusion is warranted that Th1 cells do not have a detectable role in the new model.

Acknowledgements The authors would like to acknowledge Fred Batenburg, Mariska van Etten and Tom Haaksma for experimental assistance and Dr Angela Fahey for the experiments validating the human IFN $\gamma$ effects on marmoset immune cells. This work was supported by PRIMOCID, which is part of the European Unionfunded project EUPRIM-NET. The funding source had no part in the design, interpretation or preparation of the data for publication.

Open Access This article is distributed under the terms of the Creative Commons Attribution Noncommercial License which permits any noncommercial use, distribution, and reproduction in any medium, provided the original author(s) and source are credited.

\section{References}

't Hart BA, Massacesi L (2009) Clinical, pathological, and immunologic aspects of the multiple sclerosis model in common marmosets (Callithrix jacchus). J Neuropathol Exp Neurol 68:341-355

't Hart BA, Amor S, Jonker M (2004) Evaluating the validity of animal models for research into therapies for immune-based disorders. Drug Discov Today 9:517-524 't Hart BA, Smith P, Amor S, Strijkers GJ, Blezer EL (2006) MRIguided immunotherapy development for multiple sclerosis in a primate. Drug Discov Today 11:58-66

't Hart BA, Blezer EL, Brok HP, Boon L, de Boer M, Bauer J, Laman JD (2005a) Treatment with chimeric anti-human CD40 antibody suppresses MRI-detectable inflammation and enlargement of pre-existing brain lesions in common marmosets affected by MOG-induced EAE. J Neuroimmunol 163:31-39

't Hart BA, Bauer J, Muller HJ, Melchers B, Nicolay K, Brok H, Bontrop RE, Lassmann H, Massacesi L (1998) Histopathological characterization of magnetic resonance imaging-detectable brain white matter lesions in a primate model of multiple sclerosis: a correlative study in the experimental autoimmune encephalomyelitis model in common marmosets (Callithrix jacchus). Am J Pathol 153:649-663

't Hart BA, Brok HP, Remarque E, Benson J, Treacy G, Amor S, Hintzen RQ, Laman JD, Bauer J, Blezer EL (2005b) Suppression of ongoing disease in a nonhuman primate model of multiple sclerosis by a human-anti-human IL-12p40 antibody. J Immunol 175:4761-4768

Axtell RC, de Jong BA, Boniface K, van der Voort LF, Bhat R, De Sarno P, Naves R, Han M, Zhong F, Castellanos JG, Mair R, Christakos A, Kolkowitz I, Katz L, Killestein J, Polman CH, de Waal MR, Steinman L, Raman C (2010) T helper type 1 and 17 cells determine efficacy of interferon-beta in multiple sclerosis and experimental encephalomyelitis. Nat Med 16:406-412

Bacchetti P, Deeks SG, McCune JM (2011) Breaking free of sample size dogma to perform innovative translational research. Sci Transl Med 3:1-4

Billiau A, Matthys P (2001) Modes of action of Freund's adjuvants in experimental models of autoimmune diseases. J Leukoc Biol 70:849-860

Boon L, Brok HP, Bauer J, Ortiz-Buijsse A, Schellekens MM, Ramdien-Murli S, Blezer E, van Meurs M, Ceuppens J, de Boer M, 't Hart BA, Laman JD (2001) Prevention of experimental autoimmune encephalomyelitis in the common marmoset (Callithrix jacchus) using a chimeric antagonist monoclonal antibody against human CD40 is associated with altered B cell responses. J Immunol 167:2942-2949

Brok HP, Heidt PJ, van der Meide PH, Zurcher C, Vossen JM (1993) Interferon-gamma prevents graft-versus-host disease after allogeneic bone marrow transplantation in mice. J Immunol 151:6451-6459

Brok HP, van Meurs M, Blezer E, Schantz A, Peritt D, Treacy G, Laman JD, Bauer J, 't Hart BA (2002) Prevention of experimental autoimmune encephalomyelitis in common marmosets using an anti-IL-12p40 monoclonal antibody. J Immunol 169:6554-6563

Brok HP, Uccelli A, Kerlero De Rosbo N, Bontrop RE, Roccatagliata L, de Groot NG, Capello E, Laman JD, Nicolay K, Mancardi GL, Ben-Nun A, 't Hart BA (2000) Myelin/oligodendrocyte glycoprotein-induced autoimmune encephalomyelitis in common marmosets: the encephalitogenic $\mathrm{T}$ cell epitope pMOG24-36 is presented by a monomorphic MHC class II molecule. J Immunol 165:1093-1101

Cahlin C, Korner A, Axelsson H, Wang W, Lundholm K, Svanberg E (2000) Experimental cancer cachexia: the role of host-derived cytokines interleukin (IL)-6, IL-12, interferon-gamma, and tumor necrosis factor alpha evaluated in gene knockout, tumor-bearing mice on C57 Bl background and eicosanoid-dependent cachexia. Cancer Res 60:5488-5493

Constantinescu CS, Hilliard B, Wysocka M, Ventura ES, Bhopale MK, Trinchieri G, Rostami AM (1999) IL-12 reverses the suppressive effect of the CD40 ligand blockade on experimental autoimmune encephalomyelitis (EAE). J Neurol Sci 171:60-64 
Feldmann M, Steinman L (2005) Design of effective immunotherapy for human autoimmunity. Nature 435:612-619

Gran B, Chu N, Zhang GX, Yu S, Li Y, Chen XH, Kamoun M, Rostami A (2004) Early administration of IL-12 suppresses EAE through induction of interferon-gamma. J Neuroimmunol 156:123-131

Jagessar SA, Smith PA, Blezer E, Delarasse C, Pham-Dinh D, Laman JD, Bauer J, Amor S, 't Hart B (2008) Autoimmunity Against Myelin Oligodendrocyte Glycoprotein Is Dispensable for the Initiation Although Essential for the Progression of Chronic Encephalomyelitis in Common Marmosets. J Neuropathol Exp Neurol

Jagessar SA, Heijmans N, Blezer ELA, Bauer J, Blokhuis JH, Wubben JAM, Drijfhout JW, van den Elsen PJ, Laman JD, ' $t$ Hart BA (2011, in press) Unravelling the T-cell mediated autoimmune attack on CNS myelin in a new primate EAE model induced with MOG34-56 peptide in incomplete adjuvant. European Journal of Immunolgy

Jagessar SA, Kap YS, Heijmans N, van Driel N, van Straalen L, Bajramovic JJ, Brok HP, Blezer EL, Bauer J, Laman JD, 't Hart BA (2010) Induction of progressive demyelinating autoimmune encephalomyelitis in common marmoset monkeys using MOG34-56 peptide in incomplete freund adjuvant. J Neuropathol Exp Neurol 69:372-385

Kap YS, Smith P, Jagessar SA, Remarque E, Blezer E, Strijkers GJ, Laman JD, Hintzen RQ, Bauer J, Brok HP, 't Hart BA (2008) Fast progression of recombinant human myelin/oligodendrocyte glycoprotein (MOG)-induced experimental autoimmune encepha- lomyelitis in marmosets is associated with the activation of MOG3456-specific cytotoxic T cells. J Immunol 180:1326-1337

Laman JD, 't Hart BA, Brok H, Meurs M, Schellekens MM, Kasran A, Boon L, Bauer J, Boer M, Ceuppens J (2002) Protection of marmoset monkeys against EAE by treatment with a murine antibody blocking CD40 (mu5D12). Eur J Immunol 32:22182228

Mauritz NJ, Holmdahl R, Jonsson R, Van der Meide PH, Scheynius A, Klareskog L (1988) Treatment with gamma-interferon triggers the onset of collagen arthritis in mice. Arthritis Rheum 31:1297-1304

Panitch HS, Hirsch RL, Haley AS, Johnson KP (1987) Exacerbations of multiple sclerosis in patients treated with gamma interferon. Lancet 1:893-895

Sanvito L, Constantinescu C, Gran B, 't Hart B (2010) The multifaceted role of interferon-g in central nervous system autoimmune demyelination. Open Autoimmun J 2:151-159

Segal BM, Constantinescu CS, Raychaudhuri A, Kim L, Fidelus-Gort R, Kasper LH (2008) Repeated subcutaneous injections of IL12/23 p40 neutralising antibody, ustekinumab, in patients with relapsingremitting multiple sclerosis: a phase II, double-blind, placebocontrolled, randomised, dose-ranging study. Lancet Neurol 7:796-804

Willenborg DO, Fordham SA, Staykova MA, Ramshaw IA, Cowden WB (1999) IFN-gamma is critical to the control of murine autoimmune encephalomyelitis and regulates both in the periphery and in the target tissue: a possible role for nitric oxide. J Immunol 163:5278-5286 